\title{
Direct stacking of sequence-specific nuclease-induced mutations to produce high oleic and low linolenic soybean oil
}

\author{
Zachary L. Demorest, Andrew Coffman, Nicholas J. Baltes, Thomas J. Stoddard, Benjamin M. Clasen, Song Luo,
} Adam Retterath, Ann Yabandith, Maria Elena Gamo, Jeff Bissen, Luc Mathis, Daniel F. Voytas and Feng Zhang ${ }^{*}$

\begin{abstract}
Background: The ability to modulate levels of individual fatty acids within soybean oil has potential to increase shelf-life and frying stability and to improve nutritional characteristics. Commodity soybean oil contains high levels of polyunsaturated linoleic and linolenic acid, which contribute to oxidative instability - a problem that has been addressed through partial hydrogenation. However, partial hydrogenation increases levels of trans-fatty acids, which have been associated with cardiovascular disease. Previously, we generated soybean lines with knockout mutations within fatty acid desaturase 2-1A (FAD2-1A) and FAD2-1B genes, resulting in oil with increased levels of monounsaturated oleic acid (18:1) and decreased levels of linoleic (18:2) and linolenic acid (18:3). Here, we stack mutations within FAD2-1A and FAD2$1 B$ with mutations in fatty acid desaturase $3 A(F A D 3 A)$ to further decrease levels of linolenic acid. Mutations were introduced into FAD3A by directly delivering TALENs into fad2-1 a fad2-1b soybean plants.
\end{abstract}

Results: Oil from fad2-1a fad2-1b fad3a plants had significantly lower levels of linolenic acid (2.5\%), as compared to fad2-1 a fad2-16 plants (4.7\%). Furthermore, oil had significantly lower levels of linoleic acid (2.7\% compared to $5.1 \%$ ) and significantly higher levels of oleic acid (82.2\% compared to $77.5 \%)$. Transgene-free fad2-1a fad2-1b fad3a soybean lines were identified.

Conclusions: The methods presented here provide an efficient means for using sequence-specific nucleases to stack quality traits in soybean. The resulting product comprised oleic acid levels above $80 \%$ and linoleic and linolenic acid levels below $3 \%$.

Keywords: TALEN, Genome editing, Soybean, High oleic acid, Low linolenic acid, Soybean oil, Targeted mutagenesis

\section{Background}

Soybean is an important legume crop that is valued for both its protein and oil content. Worldwide soybean production in 2014/2015 was 319 million metric tons, with 108 million metric tons produced in the United States. Soybean oil is used in applications ranging from cooking and frying to industrial lubrication and biofuels. Commodity soybean oil is primarily composed of five fatty acids: palmitic acid ( 13\%, saturated, 16:0), stearic acid ( $\sim 4 \%$, saturated, 18:0), oleic acid ( $20 \%$, monounsaturated, 18:1), linoleic acid ( 55\%, polyunsaturated, 18:2) and linolenic acid ( 8 \%, polyunsaturated, 18:3).

\footnotetext{
* Correspondence: fzhang@calyxt.com

Calyxt, Inc., 600 County Road D West Suite 8, New Brighton, MN 55112, USA
}

Due to high levels of polyunsaturated fatty acids, soybean oil has poor oxidative and frying stability, which limits its use in food products and industrial applications. In an effort to lower the levels of polyunsaturated fatty acids, soybean oil is partially hydrogenated; however, partial hydrogenation significantly increases transfatty acids, which have been linked with coronary heart disease and buildup of plaque in arteries [1]. The Food and Drug Administration (FDA) made a preliminary determination that partially hydrogenated oils are no longer 'generally recognized as safe' (GRAS) and is now taking steps to remove artificial trans fats from human food [2]. Altering the composition of soybean oil by 
decreasing the levels of polyunsaturated fatty acids may help reduce the need for hydrogenation.

Significant progress has been made in uncovering the genes involved in the soybean lipid biosynthetic pathway, and those involved in conversion of oleic acid into polyunsaturated fatty acids. Conversion of oleic to linoleic acid is catalyzed by fatty acid desaturase 2 (FAD2) proteins [3]. There are three $F A D 2$ desaturase genes within the soybean genome, FAD2-1A (Glyma10g42470), FAD2-1B (Glyma20g24530) and FAD2-2 (Glyma03g30070). Both $F A D 2-1 A$ and $F A D 2-1 B$ are highly expressed during peak oil synthesis and are the primary genetic determinants of oleic and linoleic acid levels in soybean seeds [4, 5]. Disruption or decreased expression of FAD2-1 genes results in oil with elevated oleic acid and decreased linoleic and linolenic acid [6-12]. Combination of mutations within $F A D 2-1 A$ and $F A D 2-1 B$ genes results in soybean oil with oleic acid levels $\sim 80 \%$ and linoleic and linolenic acid levels $\sim 5 \%$ each $[13,14]$.

Decreasing levels of linolenic acid is predicted to improve soybean oil characteristics by decreasing total levels of polyunsaturated fatty acids, and subsequently increasing frying and oxidative stability. Conversion of linoleic to linolenic acid is catalyzed by the fatty acid desaturase 3 (FAD3) enzyme, which is produced by a family of genes consisting of FAD3A (Glyma14g37350), FAD3B (Glyma02g39230) and FAD3C (Glyma18g06950). Consistent with its high expression in developing seeds, FAD3A has the greatest effect on linolenic acid concentrations in soybean oil [15]. Combining mutations within FAD $3 A$ with $F A D 3 B$ and/or $F A D 3 C$ resulted in oil having $<3 \%$ linolenic acid [16-20]

With the advent of sequence-specific nucleases, including TALENs and CRISPR/Cas, it has become possible to introduce targeted knockout mutations within genes of interest [21]. When delivered to plant cells, sequencespecific nucleases generate targeted DNA double-strand breaks. These double-strand breaks are then repaired predominantly by non-homologous end joining (NHEJ), which may result in the introduction of small insertions or deletions at the repair site. If double-strand breaks are generated within gene coding sequences, imprecise repair by NHEJ has potential to introduce frameshift mutations or in-frame deletions that destroy protein function. The objective of this study was to create high oleic and low linolenic soybean lines by stacking targeted mutations within FAD2-1A, FAD2-1B and FAD3 genes. With the current industrial standard for low linolenic acid soybean oil being $3 \%$ [20], we sought to inactivate a sufficient number of $F A D 3$ genes to achieve linolenic levels $<3 \%$.

\section{Results}

\section{Designing TALENs targeting the soybean FAD3 genes}

Soybean oil is primarily composed of palmitic acid, stearic acid, oleic acid, linoleic acid and linolenic acid. In oil from wild type plants, these five fatty acids are present at approximately $13,4,20,55$ and $8 \%$, respectively (Fig. 1a). Previously, we used TALENs to generate knockout mutations within both FAD2-1A and FAD2-1B genes [14]. Oil from the resulting plants contained higher levels of oleic acid ( $79 \%)$ and lower levels of

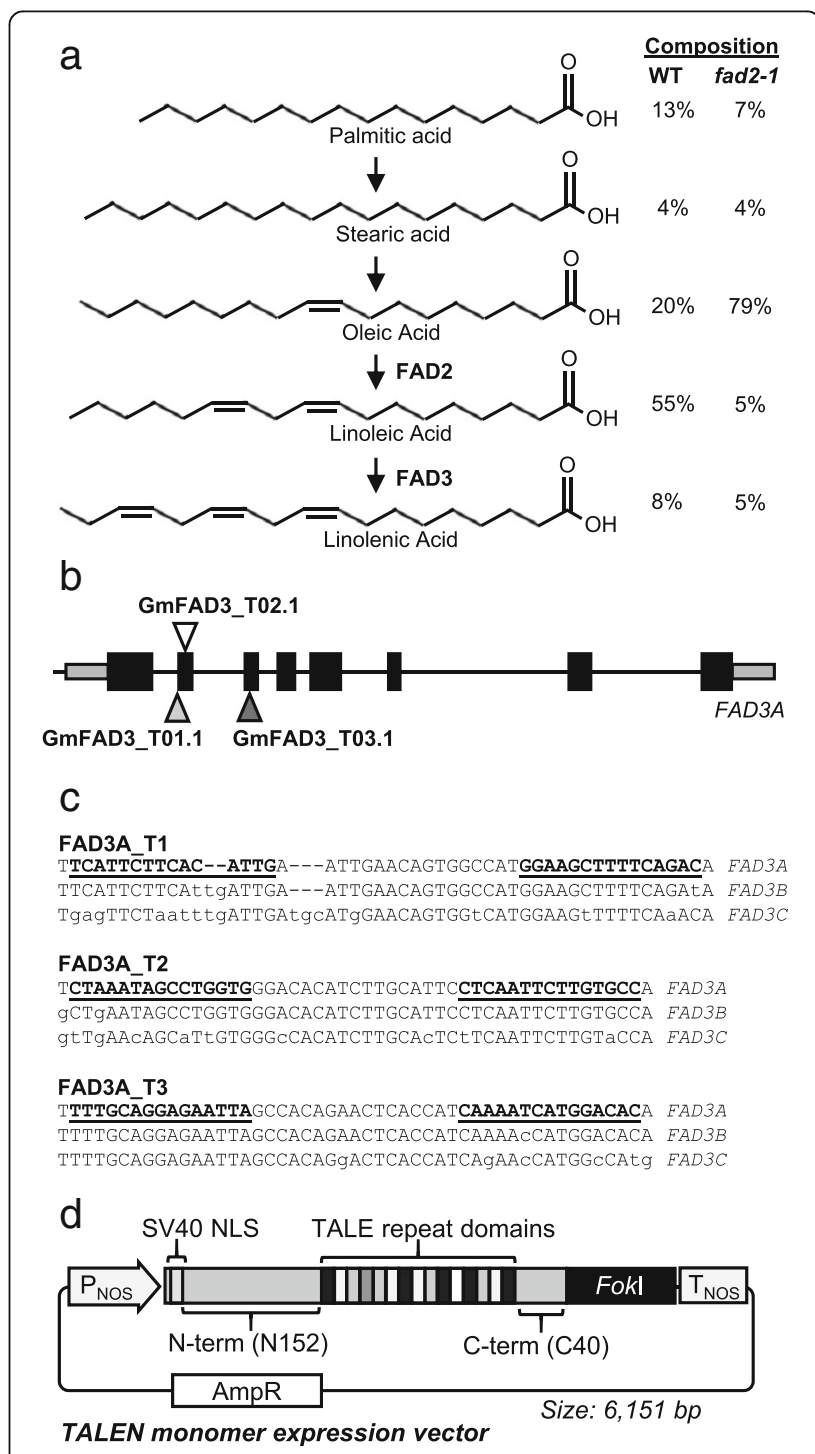

Fig. 1 Design of TALENs targeting FAD3 genes within Glycine max. a Illustration of the fatty acid pathway. Relative percent composition of individual fatty acids in the oil from WT and fad2-1 knockout plants is shown on the right. $\mathbf{b}$ Schematic of the FAD $3 A$ genomic sequence. Triangles, approximate TALEN binding sites; black boxes, exons; gray boxes, 5' and 3' untranslated regions. c Nucleotide sequences of the predicted TALEN target sites within the FAD3A, $F A D 3 B$, and FAD $3 A$ genes. Bold and underlined nucleotides indicate TALEN binding sequence. Lower case nucleotides indicate positions of SNPS. $\mathbf{d}$ Illustration of a TALEN monomer expression vector. P NOS, $_{\text {, }}$ nopaline synthase promoter; SV40 NLS, simian virus 40 large T-antigen nuclear localization signal; $T_{\text {NOS }}$, nopaline synthase terminator; $A m p R$, ampicillin resistance gene 
linoleic and linolenic acids ( $5 \%$ each), compared to oil from WT plants. Here, we sought to further improve oil characteristics by knocking out genes involved in the conversion of linoleic to linolenic acid. We predicted that by knocking out the FAD3 linoleate desaturase genes, levels of linolenic acid would further decrease.

The soybean genome contains three linoleate desaturase genes: FAD3A (Glyma14g37350), FAD3B (Glyma02g39230) and $F A D 3 C$ (Glyma18g06950). In terms of nucleotide similarity of their coding sequences, $F A D 3 A$ shares $96.2 \%$ identity to $F A D 3 B$ and $14.4 \%$ identity to $F A D 3 C$. Compared to $F A D 3 B$ and $F A D 3 C$, mutations in FAD $3 A$ confer the greatest decrease in linolenic acid levels in soybean oil (from $\sim 8$ to $\sim 4 \%$ ) [22], which is consistent with higher expression of FAD3A within developing seeds [15]. Therefore, we sought to design TALENs that primarily recognize FAD3A sequence. Three TALEN pairs were synthesized which recognize sequence within exon two or exon three of FAD3A (designated as GmFAD3_T01.1, GmFAD3_T02.1, and GmFAD3_T03.1) (Fig. 1b). TALENs were designed to recognize $F A D 3 A$ sequence which is partially conserved between $F A D 3 B$ and $F A D 3 C$; however, the recognition sequences for all TALEN pairs at $F A D 3 B$ and $F A D 3 C$ contained at least one single-nucleotide polymorphism (SNP), but up to 11 SNPs, when compared to the FAD3A sequence (Fig. 1c).

\section{Assessing TALEN activity in protoplasts by deep- sequencing}

To determine TALEN activity, protoplasts were transformed with plasmid DNA encoding each TALEN pair and the FAD3 target sites were deep-sequenced. To this end, approximately 500000 protoplasts were transformed with $15 \mu \mathrm{g}$ each of two plasmids encoding a complete TALEN pair. Protoplasts were transformed using polyethylene glycol. Genomic DNA was isolated $\sim 48 \mathrm{~h}$ post transformation and used as a template in a PCR with primers designed to individually amplify TALEN target sites within the $F A D 3 A, F A D 3 B$ or $F A D 3 C$ gene. Amplicon pools for each TALEN target site were sequenced by 454 pyrosequencing. For all three TALEN pairs, we observed evidence of NHEJ mutations in two of the three FAD3 genes (Fig. 2a). TALEN pair GmFAD3_T02.1 introduced mutations within both FAD3A and FAD3B, and, relative to the other TALEN pairs, had the highest activity at its intended target sequence, FAD3A (16.0\%). On the other hand, TALEN pair GmFAD3_T03.1 had the lowest activity at its intended FAD $3 A$ target sequence (4.9\%). Activity of all three TALEN pairs at the $F A D 3 B$ and $F A D 3 C$ target sites was lower than the respective $F A D 3 A$ target site, which is most likely due to SNPs within the $F A D 3 B$ and FAD $3 C$ TALEN binding sites.

We observed a correlation between the number of SNPs within TALEN binding sites and the relative mutation

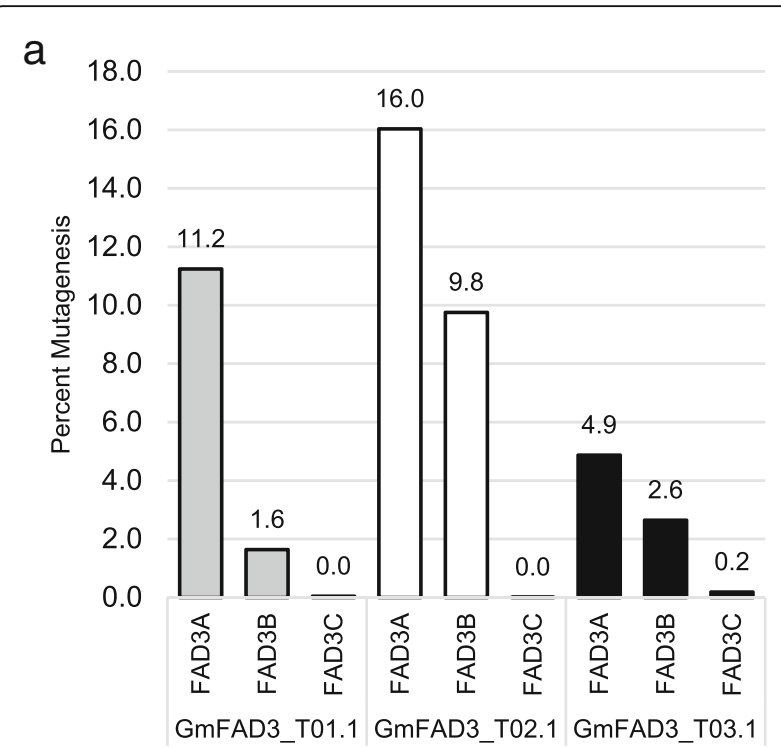

b

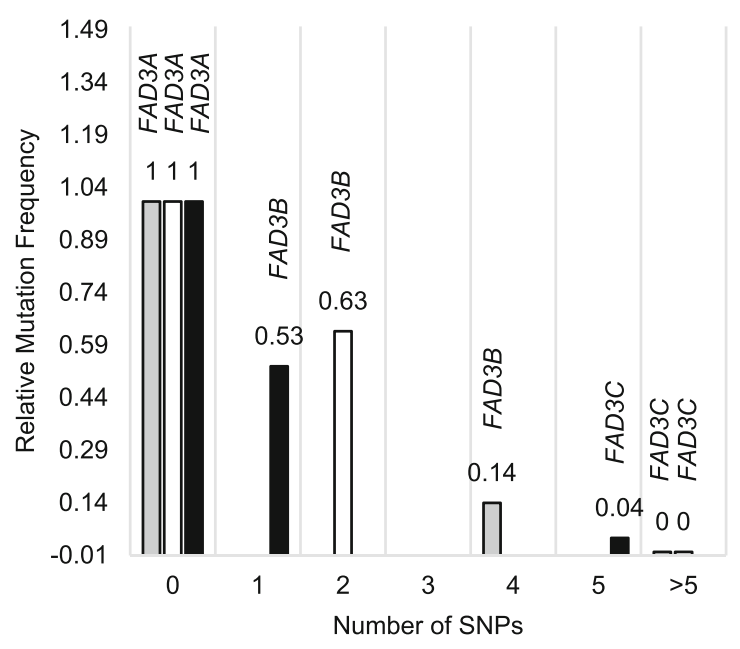

口GmFAD3_T01.1 घGmFAD3_T02.1 घGmFAD3_T03.1

Fig. 2 FAD3 TALEN activity in soybean protoplasts. a TALEN pairs were assessed for their activity $\sim 48 \mathrm{~h}$ after transformation in soybean protoplast. The frequency of mutagenesis represents the total number of sequence reads with insertions or deletions divided by the total number of sequence reads. The resulting number was then divided by the transformation frequency ( $90 \%$ ) which was determined using a YFP control plasmid. b TALEN activity relative to the number of SNPs present within the predicted TALEN binding sites

frequencies (Fig. 2b). Mutation frequencies at $F A D 3 A$ target sites (containing 0 SNPs) for TALEN pairs GmFAD3_T01.1, GmFAD3_T02.1, GmFAD3_T03.1 were 11.2, 16.0 and $4.9 \%$ respectively. After normalizing TALEN mutation frequencies at $F A D 3 A$, the relative mutation frequencies at $F A D 3 B$ and $F A D 3 C$ were determined. Target sites with one or two SNPs decreased mutation frequencies to $\sim 53$ or $63 \%$, respectively, relative to the activity of the corresponding TALEN FAD $3 A$; target 
sites with four SNPs decreased mutation frequencies to $14 \%$; target sites with five SNPs decreased mutation frequencies to $0.041 \%$, and target sites with $>5$ SNPs decreased mutation frequencies to undetectable levels. Whereas these data do not account for relative position of the SNPs, they provide evidence for TALEN target site specificity, indicating that target sites with five or more SNPs are unlikely to be recognized and cleaved.

\section{Generating soybean plants with FAD3 mutations}

To generate soybean plants with knockout mutations in FAD3 genes, DNA encoding TALEN pair GmFAD3_T02.1 was stably integrated into the soybean genome [14, 23]. Both WT and fad2-1a fad2-1b mutant soybean lines were transformed; from four independent transformations, a total of 72 events were generated (Table 1). To detect TALEN-induced mutations, the FAD3A gene was amplified and digested with T7 endonuclease I. We observed that 16 of the 72 events had cleavage products consistent with mutations within the GmFAD3_T02.1 target sequence. Cloning and sequencing of FAD3A amplicons revealed that all 16 plants harbored short deletions within the TALEN spacer sequence, ranging from 4 to $135 \mathrm{bp}$. Together, these results confirm the successful mutagenesis of $F A D 3 A$ within T0 soybean plants, with a mutagenesis frequency of $\sim 22 \%$.

To confirm TALEN-induced mutations can be stably transmitted to subsequent generations, candidate T1 plants derived from experiment Gm183 were screened for mutations within FAD $3 A$ by PCR amplification and sequencing of clones. From three different T0 events (Gm183-4, Gm183-5 and Gm183-6), we identified T1 plants harboring heterozygous or homozygous mutations within $F A D 3 A$, indicating that mutations were stably transmitted to the next generation (Table 2). Further, we assessed T1 plants by PCR for the presence of transgene sequence. Of the $25 \mathrm{~T} 1$ plants assayed, 20 were positive for transgene sequence and five were negative (i.e., null segregant for the TALEN transgene). Importantly, two of the five transgene-free T1 plants harbored mutations within $F A D 3 A$. These two

Table 1 Summary of FAD3A mutation frequencies within T0 soybean plants

\begin{tabular}{lllll}
\hline Experiment & Background & $\begin{array}{l}\text { Number } \\
\text { of events }\end{array}$ & $\begin{array}{l}\text { Number of } \\
\text { T7-positive } \\
\text { events }\end{array}$ & $\begin{array}{l}\text { Mutagenesis } \\
\text { frequency }\end{array}$ \\
\hline Gm183 & fad2-1a fad2-16 & 30 & 8 & $27 \%$ \\
Gm184 & Bert & 35 & 5 & $14 \%$ \\
Gm205 & fad2-1a fad2-1b & 3 & 2 & $67 \%$ \\
Gm206 & Bert & 4 & 1 & $25 \%$ \\
Average & & 72 & 16 & $22 \%$ \\
mutagenesis & & & & \\
\hline
\end{tabular}

Table 2 Genotype of T1 plants from candidate T0 events harboring mutations within FAD3A

\begin{tabular}{|c|c|c|c|}
\hline Parent line (T0) & T1 plant number & $\begin{array}{l}\text { FAD3A } \\
\text { genotype }\end{array}$ & $\begin{array}{l}\text { Presence of } \\
\text { transgene }\end{array}$ \\
\hline \multirow[t]{4}{*}{ Gm183-4 } & 1 & $-7 \mathrm{bp} / \mathrm{WT}$ & Undetected \\
\hline & 2 & $-7 \mathrm{bp} /-7 \mathrm{bp}$ & + \\
\hline & 3 & $-7 \mathrm{bp} /-7 \mathrm{bp}$ & + \\
\hline & 4 & $-7 \mathrm{bp} / \mathrm{WT}$ & + \\
\hline \multirow[t]{7}{*}{ Gm183-5 } & 2 & $-43 \mathrm{bp} / \mathrm{WT}$ & + \\
\hline & 3 & WT/WT & Undetected \\
\hline & 4 & $-43 b p /-43 b p$ & + \\
\hline & 5 & $-43 b p /-43 b p$ & Undetected \\
\hline & 7 & $-43 b p /-43 b p$ & + \\
\hline & 8 & $-4 \mathrm{bp} /-4 \mathrm{bp}$ & + \\
\hline & 9 & $-43 b p /-43 b p$ & + \\
\hline \multirow[t]{14}{*}{ Gm183-6 } & 1 & $-4 \mathrm{bp} /-4 \mathrm{bp}$ & + \\
\hline & 2 & WT/WT & + \\
\hline & 3 & WT/NT & + \\
\hline & 4 & $-4 \mathrm{bp} / \mathrm{WT}$ & + \\
\hline & 5 & $-4 \mathrm{bp} /-4 \mathrm{bp}$ & + \\
\hline & 6 & WT/WT & Undetected \\
\hline & 7 & $-4 \mathrm{bp} / \mathrm{WT}$ & + \\
\hline & 8 & WT/WT & Undetected \\
\hline & 9 & WT/WT & + \\
\hline & 10 & WT/WT & + \\
\hline & 11 & $-4 \mathrm{bp} / \mathrm{WT}$ & + \\
\hline & 12 & $-4 \mathrm{bp} / \mathrm{WT}$ & + \\
\hline & 13 & WT/WT & + \\
\hline & 14 & $-4 \mathrm{bp} / \mathrm{WT}$ & + \\
\hline
\end{tabular}

plants were self-pollinated to produce homozygousmutant, transgene-free $f a d 2-1 a$ fad2-1b fad $3 a$ soybean plants. Notably, we also identified a single-gene fad $3 a$ knockout T1 plant from experiment Gm184 (identified as Gm184-3-20) which contains a homozygous -4 bp deletion within FAD3A. We failed, however, to identify plants with combinations of FAD3A and $F A D 3 B$ mutations, indicating that the frequency of mutagenesis at $F A D 3 B$ was $<1.4 \%$ (i.e., less than 1 out of 72 events).

\section{Oil from fad2-1a fad2-16 fad3a homozygous mutant soybean seeds contains high oleic, low linoleic and low linolenic acid}

Next, we assessed the oil profile within seed from fad $3 a$ and fad2-1a fad2-1b fad3a homozygous mutant soybean lines (Fig. 3). Seed from T1 homozygous mutant lines were collected and assessed for oil composition by gas chromatographic analysis of fatty 


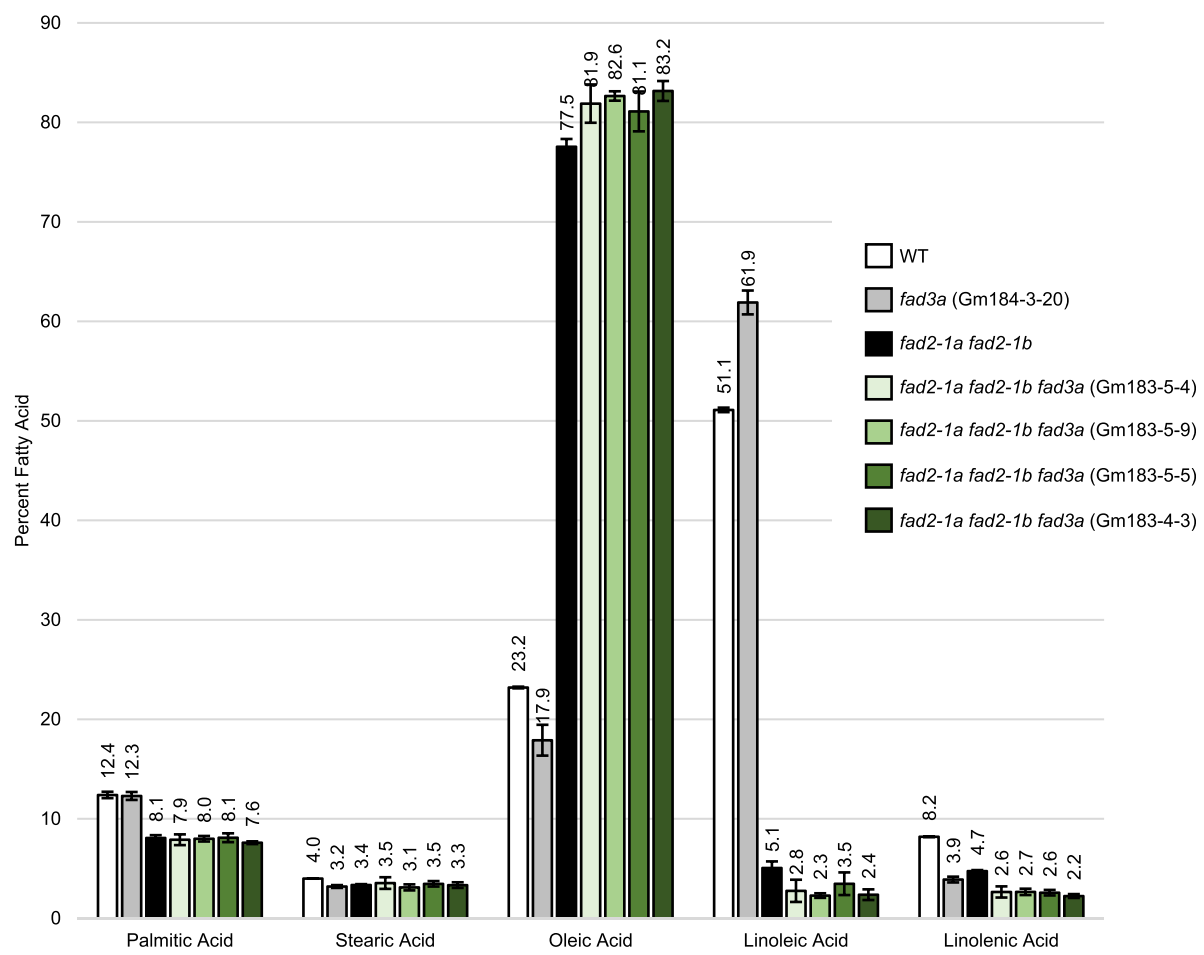

Fig. 3 Fatty acid profile from fad2-1 a fad2-1b fad3a soybean plants. Oil from T2 seed from four different T1 fad2-1a fad2-1b fad $3 a$ mutant lines was analyzed. The genotypes for the fad2-1 a fad2-1b fad3a plant lines at the fad3a TALEN target site were $-7 \mathrm{bp} /-7 \mathrm{bp}(\mathrm{Gm} 183-4-3)$, -43 bp/$43 \mathrm{bp}$ (Gm183-5-4), -43 bp/-43 bp (Gm183-5-5), and -43 bp/-43 bp (Gm183-5-9). The genotype for the fad3a plant line was -4 bp/-4 bp (Gm184-3-20). Error bars represent standard deviation of the oil levels within individual seeds, specifically, five seeds for Gm183-4-3, five seeds for Gm183-5-4, five seeds for Gm183-5-5, five seeds for Gm183-5-9, five seeds for Gm184-3-20, four seeds for WT, and 20 seeds for fad2-1a fad2-1b

acid methyl esters (GC FAME Analysis; Additional file 1). In oil from fad3a plants, we observed significant changes in linolenic, linoleic, oleic and stearic acid levels, relative to oil from WT plants. We observed linolenic acid decreased from $8.2 \pm 0.4$ to 3.9 $\pm 0.3 \%$, linoleic acid increased from $51.1 \pm 0.2$ to $61.9 \pm 1.2 \%$, oleic acid decreased from $23.2 \pm 0.8$ to $17.9 \pm 1.6 \%$ and stearic acid decreased from $4 \pm 0.01$ to $3.2 \pm 0.1 \%$.

We observed significant changes in fatty acid levels within seed oil from fad2-1a fad2-1b fad3a soybean plants, when compared to fad2-1a fad2-1b soybean plants. The average linolenic acid level within oil from fad2-1a fad2-1b fad $3 a$ plants was $2.5 \pm 0.4 \%$, significantly lower than oil from fad2-1a fad2-1b soybean plants $(4.7 \pm 0.1 \%)$. Linoleic acid levels decreased from $5.1 \pm 0.7 \%$ in fad2-1a fad $2-1 b$ lines to $2.7 \pm 0.9 \%$ in fad2-1a fad2-1b fad3a lines, and oleic acid levels increased from $77.5 \pm 0.8 \%$ in fad2-1a fad2-1b lines to $82.2 \pm 1.6 \%$ in fad2-1a fad $2-1 b$ fad $3 a$ lines. Together, these results indicate that stacking mutations within $F A D 2-1$ and FAD3A genes decreases linolenic and linoleic acid levels to below $3 \%$, and increases oleic acid levels to over $80 \%$.

\section{Discussion}

In 2015, the FDA ruled that trans fat is no longer 'generally recognized as safe' for use in food, and has set a 3 year deadline to remove partially hydrogenated oils from food products. In an effort to improve shelf life and cooking characteristics, soybean oil is partially hydrogenated. However, partial hydrogenation results in increased levels of trans fats. Generating soybean oil with lower levels of polyunsaturated fatty acids promises to enhance shelf life and heat stability, thereby reducing the need for hydrogenation. Previously, we generated soybean that produce high oleic acid oil by knocking out both FAD2-1A and FAD2-1B genes. Here, we further improved oil characteristics by decreasing polyunsaturated fatty acids (linoleic and linolenic) to levels below $3 \%$. The methods and products presented here provide solutions for the demand of soybean oil with increased oxidative stability.

Soybean plants with high oleic acid have been developed by research groups using different approaches. These approaches include RNAi [11], combining naturally occurring or induced mutations [13, 24, 25], and site-directed mutagenesis using sequence-specific nucleases [14]. Of the characterized high oleic soybean lines, most have 
distinct differences in the levels of individual fatty acids. For example, Monsanto's Vistive ${ }^{\bullet}$ Gold high oleic acid soybean oil (MON 87705), created using RNAi targeting $F A T B$ and FAD2-1 and harboring naturally occurring mutations in FAD3 genes, has oleic, linoleic, linolenic and saturated fatty acids levels of 71.7, 16.9, 2.9 and $6.8 \%$, respectively [26]. Further, Pioneer/DuPont's Plenish ${ }^{\circ}$ high oleic soybean oil (HOSO 305423), created using RNAi against FAD2-1, has oleic, linoleic, linolenic and saturated fatty acids levels of 70.6, 5.5, 7.2 and $14.3 \%$ respectively $[26,27]$. The fad2-1a fad2-1b fad $3 a$ lines created here have oleic, linoleic, linolenic and saturated fatty acids levels of $82.2,2.7,2.5$, and $11.3 \%$, respectively. Variations in fatty acid levels may be due to differences in gene targets (e.g., FATB vs $F A D 3$ ), genetic background, growth conditions, and possibly incomplete silencing of gene expression when using RNAi technology.

We observed lower levels of linoleic acid and higher levels of oleic acid within oil from fad2-1a fad2-1b fad3a plants, when compared to oil from fad2-1a fad2-1b plants. It would be expected that knockout mutations within desaturase genes would result in accumulation of the corresponding substrate. Indeed, this is the case for plants containing mutations in either FAD3A or FAD2$1 A F A D 2-1 B$; mutations in $F A D 3 A$ resulted in increased levels of linoleic acid, and mutations in FAD2-1A FAD2$1 B$ resulted in increased levels of oleic acid. When we introduced FAD3A mutations within fad2-1a fad2-1b soybeans, the level of linoleic acid decreased and the levels of oleic acid increased. This trend was also observed in high oleic and low linolenic soybean plants generated after combining different sources of mutant $F A D 2-1 A, F A D 2-1 B$ and FAD3A genes [20]; however, fatty acids levels were significantly affected by environmental conditions. Further, and unexpectedly, we observed that oil within fad3a and fad2-1a fad2-1b plants had deceased levels of the two fatty acids immediately preceding the substrate of the inactivated desaturase (i.e., palmitic and stearic acid in fad2-1a fad2-1b plants, or stearic and oleic acid in fad $3 a$ plants). Understanding properties of additional soybean desaturase proteins and the effects of genetic background and environmental conditions may provide a better understanding of the lipid biosynthetic pathway in soybean.

Here, we used TALENs to generate $f a d 2-1 a$ fad2- $1 b$ fad3 knockout soybean lines; however, there are other sequence-specific nucleases that can be used for plant genome editing, including meganucleases, zinc-finger nucleases and CRISPR/Cas systems. Three key parameters for choosing a sequence-specific nuclease include efficacy (i.e., how likely will the nuclease introduce a desired modification), target site specificity, and ease of construction. Although meganucleases and zinc-finger nucleases have achieved acceptable mutation frequencies and target site specificity $[28,29]$, their widespread use has been hindered due to difficulties with construction [30, 31]. TALEN and CRISPR/Cas systems have overcome these challenges as they have a modular 'one RVD to one base pair' design or a RNA-DNA interaction, allowing for efficient reconstruction of nucleases with altered target sites. One difference between TALENs and CRISPR/Cas is target site length. CRISPR/Cas9 from Streptococcus pyogenes recognizes, in general, 17-20 nucleotides of sequence plus a three nucleotide PAM sequence (NGG), providing 19-22 nucleotides of target site specificity. TALEN pairs, on the other hand, are frequently engineered to recognize 30-40 nucleotides, which may lead to fewer off-target double-strand breaks. Whereas both TALENs and CRISPR/Cas9 tolerate certain nucleotide changes within their target sequences, both provide sufficient specificity to target a single site within a complex plant genome, provided the target site (or a similar target site) is not repeated elsewhere in the genome.

An advantage of engineering crops with sequencespecific nucleases is that the resulting product is not required to harbor transgenic DNA. Within this study, we identified two modified soybean lines with undetectable levels of transgenic DNA. The genotype of these plants were described to the USDA for the purpose of determining regulatory status. An opinion letter, issued May 20th, 2015, indicated that the resulting FAD3A knockout plants are not regulated by the USDA under seven CFR part 340 [32]. This means that trials can be launched with transgene-free $f a d 3 a$ knockout plants to assess their phenotype in field grown conditions. Due to the lengthy and costly deregulation process, the technology and methods presented within this study provide a clear advantage over conventional transgenesis, thereby enabling more groups to contribute to crop improvement and food security.

\section{Conclusions}

Here we describe methods to efficiently stack quality traits within plants using sequence-specific nucleases. TALENs targeting FAD3 were directly delivered to soybean fad2-1a and $f a d 2-1 b$ knockout lines to produce triple knockout fad2-1a fad2-1b fad3 plants. Seed oil from the triple knockout lines had significantly altered fatty acid levels, compared to the parent fad2-1a fad2-1b lines. The polyunsaturated fatty acids, linoleic and linolenic acid, decreased to levels below $3 \%$, and the monounsaturated fatty acid oleic acid increased to levels over $80 \%$.

\section{Methods}

Plant material

Plant material used within this study was from soybean [Glycine max (L.) Merr.] variety 'Bert'. 


\section{Plasmid construction}

Coding sequences for the TALEN pairs used in this study (GmFAD3_T01.1, GmFAD3_T02.1, and GmFAD3_T03.1) were synthesized as previously described [33]. Individual TALEN monomers were cloned into protoplast expression vectors harboring a nopaline synthase (NOS) promoter and terminator. TALEN backbone architecture comprised N-terminal truncations (N152: TAAAKFERQHMDSID IADLRTLGYSQQQQEKIKPKVRSTVAQHHEALVGHGF THAHIVALSQHPAALGTVAVKYQDMIAALPEATHEAI VGVGKQWSGARALEALLTVAGELRGPPLQLDTGQLL KIAKRGGVTAVEAVHAWRNALTGAPLN) and C-ter minal truncations (C40: SIVAQLSRPDPALAALTNDHLV ALACLGGRPALDAVKKGL). Each TALEN monomer comprised 15 repeat domains for targeting 15 nucleotides of FAD3 sequence, as shown in Fig. 1c. Repeat variable diresidues within the TALE repeats included NI (for targeting adenine), HD (for targeting cytosine), NN (for targeting guanine), and NG (for targeting thymine). To facilitate trafficking to plant cell nuclei, an SV40 NLS (PKKKRKV) was added to the N-terminus of the TALEN protein. The size of plasmids encoding TALE monomers was $6151 \mathrm{bp}$. Plasmids were isolated from bacteria using the QIAGEN $^{\circ}$ maxiprep kit.

\section{Soybean transformation}

Experiments within this study were performed using the soybean variety, 'Bert', and the fad2-1a fad2-1b double homozygous mutant soybean line as previously described [14]. Transformation was carried out using following previously described protocols $[14,23]$. Briefly, half-seeds were transformed with plasmid sequence encoding TALEN pairs and a selectable marker, and soybean plants were regenerated on medium containing glufosinate [34]. Explants were incubated in a growth incubator at $28{ }^{\circ} \mathrm{C}$ with $\sim 110 \mu \mathrm{mol} / \mathrm{m}^{2} / \mathrm{s}$ of light. Rooted seedlings were transferred to soil containing a peatbased substrate (BM1, Berger, Les Tourbièr Berger Ltee, Saint-Modeste, QC, Canada), and acclimated to ambient humidity.

\section{Protoplast transformation}

TALEN pairs were assessed for activity using soybean protoplasts. Protoplasts were isolated from immature cotyledons similar to previously described protocols [35]. Briefly, immature cotyledons were digested in an enzyme solution containing $0.45 \mathrm{M}$ D-mannitol, $20 \mathrm{mM}$ MES, $2 \%$ cellulose, $0.5 \%$ macerozyme, $\mathrm{pH}$ 5.8. Digestion was carried out for $16 \mathrm{~h}$ at $25{ }^{\circ} \mathrm{C}$ in the dark with shaking at $26 \mathrm{rpm}$. Protoplasts were passed through a $100 \mu \mathrm{m}$ cell filter and collected in a $50 \mathrm{~mL}$ Falcon tube. Protoplasts were then pelleted by centrifugation at $100 \mathrm{rpm}$ for $5 \mathrm{~min}$. Supernatant was removed and cells were resuspended in WB-N solution (0.45 M D-mannitol, $10 \mathrm{mM}$ calcium chloride, $\mathrm{pH}$ 5.8). Protoplasts were transformed using polyethylene glycol 4000 (20\% diluted concentration) for $30 \mathrm{~min}$. For each TALEN pair, 500 000 protoplasts were transformed with $30 \mu \mathrm{g}$ of plasmid $(15 \mu \mathrm{g}$ for each TALEN pair). Protoplasts were washed three times in WB-N and transferred to low retention $15 \times 10 \mathrm{~mm}$ petri plates. Protoplasts were incubated at $25{ }^{\circ} \mathrm{C}$ for $48 \mathrm{~h}$ before genomic DNA was isolated.

\section{Genotyping and 454 pyrosequencing}

To assess TALEN activity by 454 pyrosequencing, and to determine the genotype of candidate FAD3 mutant plants, the FAD3A, FAD3B and/or FAD3C TALEN target sequences were individually amplified by PCR. Primers for amplifying the FAD3A gene were GmFAD3A_F1 (5'ACACTGCTTTGTTATGCCTACCTCAT) and GmFAD3 A_R1 (5'-CTTCTCGGTTAACTAAGATAATGACAAAA AAAAATG). Primers for amplifying the $F A D 3 B$ gene were GmFAD3B_F1 (5'-TCTCACACATTGTTCTGTTATGTC ATTTCTTC) and GmFAD3B_R1 (5'- GTTAACTAAGAT AATGACACATAAAAAAGAGCCATG). Primers for am plifying the $F A D 3 C$ gene were GmFAD3C_F1 (5'- GGAC ATGATTGGTAACTAATTATTATTACAAATTGTTATG TTATGTTATG) and GmFAD3C_R1 (5'-CAAAGATGGG GAAAGGAAGAGTGAATC).

\section{Oil analysis}

Individual T2 seeds from homozygous-mutant fad2-1a $f a d 2-1 b$ fad $3 a$ T1 plants were isolated and assessed for oil composition, as shown in Fig. 3. Five T2 seeds from each of four different T1 parent plants with a fad2-1a $f a d 2-1 b$ fad $3 a$ genotype were sampled; 20 seeds from fad2-1a fad2-1b lines were sampled, five seeds from one fad $3 a$ parent was sampled, and four WT seeds were sampled. Seeds were sent to Eurofins BioDiagnostics (507 Highland Drive, River Falls, WI 54022) for fatty acid analysis. Oil composition was determined using gas chromatographic analysis of fatty acid methyl esters (GC FAME Analysis). The fatty acid levels were reported as the percentage of palmitic, stearic, oleic, linoleic, and linolenic acids to the total fatty acids. Raw GC FAME data is presented in Additional file 1.

\section{Additional file}

Additional file 1: GC FAME data contains the raw GC FAME data used to generate the graph in Fig. 3. (XLSX $18 \mathrm{~kb}$ )

\section{Abbreviations}

FAD2: Fatty acid desaturase 2; FAD3: Fatty acid desaturase 3; FDA: Food and drug administration; GC FAME: Gas chromatographic analysis of fatty acid methyl esters; GRAS: Generally recognized as safe; NHEJ: Non-homologous end joining; NOS: Nopaline synthase; SNP: Single nucleotide polymorphism; TALEN: Transcription activator-like effector nuclease 


\section{Acknowledgments}

Not applicable.

\section{Funding}

Funding for the research reported was provided by Cellectis SA. The funding body did not play a role in the design of the study, collection, analysis or interpretation of the data, or in the writing of the manuscript.

\section{Availability of data and material}

Data supporting the results of this article are included within the article and additional files. The GC FAME data is included within Additional file 1.

\section{Authors' contributions}

ZLD designed the TALEN reagents and prepared soybean seeds for oil analysis. AY, MEG and JB constructed the TALEN plasmids, prepared 454 pyrosequencing samples, prepared soybean seeds for oil analysis and genotyped the soybean plants. NJB, ZLD and TJS wrote the manuscript. ZLD and NJB analyzed the data. ZLD, SL, LM, DFV and FZ conceived the experiments. AC performed the soybean transformations. BMC and AR maintained the soybean plants. All authors have read and approved the final version of the manuscript.

\section{Competing interests}

All authors are employees of Cellectis SA. Calyxt, Inc. is a subsidiary of Cellectis SA.

\section{Consent for publication}

Not applicable.

\section{Ethics approval and consent to participate} Not applicable.

\section{Received: 29 April 2016 Accepted: 26 September 2016}

Published online: 13 October 2016

\section{References}

1. Ascherio A, Katan M, Zock P, Stampfer M, Willett W. Trans fatty acids and coronary heart disease. N Engl J Med. 1999;340:1994-8.

2. Unnevehr $L$, Jagmanaite $E$. Getting rid of trans fats in the US diet: policies, incentives and progress. Food Policy. 2008:33:497-503.

3. Okuley J, Lightner J, Feldmann K, Yadav N, Lark E, Browse J. Arabidopsis FAD2 gene encodes the enzyme that is essential for polyunsaturated lipid synthesis. Plant Cell. 1994;6:147.

4. Miquel MF. High-oleate oilseeds fail to develop at low temperature. Plant Physiol. 1994;106:421-7.

5. Heppard EP, Kinney AJ, Stecca KL, Miao GH. Developmental and growth temperature regulation of two different microsomal [omega]-6 desaturase genes in soybeans. Plant Physiol. 1996;110:311-9.

6. Park H, Graef $G, X u Y$, Tenopir $P$, Clemente TE. Stacking of a stearoyl-ACP thioestersae with a dual-silenced palmitoyl-ACP thioesterase and $\Delta 12$ fatty acid desaturase in transgenic soybean. Plant Biotechnol J. 2014;12:1035-43.

7. Alt JL, Fehr WR, Welke GA, Sandhu D. Phenotypic and molecular analysis of oleate content in the mutant soybean line M23. Crop Sci. 2005:45:1997-2000.

8. Sandhu D, Alt JL, Scherder CW, Fehr WR, Bhattacharyya MK. Enhanced oleic acid content in the soybean mutant M23 is associated with the deletion in the Fad2-1a gene encoding a fatty acid desaturase. J Am Oil Chem Soc. 2007;84:229-35.

9. Zhang L, Yang XD, Zhang YY, Yang J, Qi GX, Guo DQ, et al. Changes in oleic acid content of transgenic soybeans by antisense RNA mediated posttranscriptional gene silencing. Int J Genomics. 2014.

10. Wagner N, Mroczka A, Roberts PD, Schreckengost W, Voelker T. RNAi trigger fragment truncation attenuates soybean FAD2-1 transcript suppression and yield intermediate oil phenotypes. Plant Biotechnol J. 2010;9:723-8.

11. Buhr T, Sato S, Ebrahim F, Xing A, Zhou Y, Mathiesen M, et al. Ribozyme termination of RNA transcripts down-regulate seed fatty acid genes in transgenic soybean. Plant J. 2002;30:155-63.

12. Graef G, LaVallee BJ, Tenopir P, Tat ME, Schweiger BJ, Kinney AJ, et al. A high oleic acid and low palmitic acid soybean: Agronomic performance and evaluation as a feedstock for biodiesel. Plant Biotechnol J. 2009;7:411-21.

13. Pham AT, Lee JD, Shannon JG, Bilyeu KD. Mutant alleles of FAD2-1A and FAD2-1B combine to produce soybeans with the high oleic acid seed oil trait. BMC Plant Biol. 2010;10:195.
14. Haun W, Coffman A, Clasen BM, Demorest ZL, Lowy A, Ray E, et al. Improved soybean oil quality by targeted mutagenesis of the fatty acid desaturase 2 gene family. Plant Biotechnol J. 2014;12:934-40.

15. Bilyeu KD, Palavalli L, Sleper DA, Beuselinck PR. Three microsomal omega-3 fatty-acid desaturase genes contribute to soybean linolenic acid levels. Crop Sci. 2003:43:1833-8

16. Bilyeu K, Palavalli L, Sleper D, Beuselinck P. Mutations in soybean microsomal omega-3 fatty acid desaturase genes reduce linolenic acid concentration in soybean seeds. Crop Sci. 2005;45:1830-6.

17. Bilyeu K, Palavalli L, Sleper DA, Beuselinck P. Molecular genetic resources for development of 1 \% linolenic acid soybeans. Crop Sci. 2006:46:1913-8.

18. Bilyeu K, Gillman JD, LeRoy AR. Novel mutant allele combinations produce soybeans containing 1 \% linolenic acid in the seed oil. Crop Sci. 2011;51:259-64.

19. Reinprecht Y, Luk-Labey SY, Larsen J, Poysa WW, Yu K, Rajcan I, et al. Molecular basis of the low linolenic acid trait in soybean EMS mutant line RG10. Plant Breed. 2009;128:253-8.

20. Pham AT, Shannon JG, Bilyeu KD. Combinations of mutant FAD2 and FAD3 genes to produce high oleic acid and low linolenic acid soybean oil. Theor Appl Genet. 2012;125:503-15.

21. Mahfouz MM, Piatek A, Stewart CN. Genome engineering via TALENs and CRISPR/Cas9 systems: challenges and perspectives. Plant Biotechnol J. 2014; 12:1006-14.

22. Chappell AS, Bilyeu KD. The low linolenic acid soybean line PI 361088B contains a novel GmFAD3A mutation. Crop Sci. 2007:47:1705-10.

23. Rech EL, Vianna GR, Aragao FJ. High-efficiency transformation by biolistics of soybean, common bean and cotton transgenic plants. Nature Protoc. 2008; 3:410-8

24. Anai T, Yamada T, Hideshima R, Kinoshita T, Rahman SM, Takagi Y. Two higholeic-acid soybean mutants, M23 and KK21, have disrupted microsomal omega6 fatty acid desaturase, encoded by GmFAD2-1a. Breed Sci. 2008;58:447-52.

25. Dierking EC, Bilyeu KD. New sources of soybean seed meal and oil composition traits identified through TILLING. BMC Plant Biol. 2009;9:89.

26. USDA-APHIS. Monsanto improved fatty acid profile MON 87705 soybean petition 09-201p: final environment assessment. Riverdale: US Department of Agriculture, Animal and Plant Health Inspection Service, Biotechnology Regulatory Services; 2011.

27. Delaney B, Appenzeller LM, Munley SM, Hoban D, Sykes GP, Malley LA, et al. Subchronic feeding study of high oleic acid soybeans (event DP-3Ø5423-1) in Sprague-Dawley rats. Food Chem Toxicol. 2008:46:3808-17.

28. Gao H, Smith J, Yang M, Jones S, Djukanovic V, Nicholson MG, et al. Heritable targeted mutagenesis in maize using a designed endonuclease. Plant J. 2010;61:176-87.

29. Townsend JA, Wright DA, Winfrey RJ, Fu F, Maeder ML, Joung JK, et al. High-frequency modification of plant genes using engineered zinc-finger nucleases. Nature. 2009;459:442-5.

30. Ramirez CL, Foley JE, Wright DA, Müller-Lerch F, Rahman SH, Cornu Tl, et al. Unexpected failure rates for modular assembly of engineered zinc fingers. Nat Methods. 2008:5:374-5.

31. Seligman LM, Chisholm KM, Chevalier BS, Chadsey MS, Edwards ST, Savage $\mathrm{H}$, et al. Mutations altering the cleavage specificity of a homing endonuclease. Nucleic Acids Res. 2002;30:3870-9.

32. USDA-APHIS. Inquiry from Cellectis Plant Sciences regarding the regulatory status of FAD3KO soybean; APHIS BRS Response to Cellectis Plant Sciences. Riverdale: US Department of Agriculture, Animal and Plant Health Inspection Service, Biotechnology Regulatory Services; 2015.

33. Beurdeley M, Bietz F, Li J, Stoddard T, Juillerat A, Zhang F, et al. Compact designer TALENs for efficient genome engineering. Nat Commun. 2013:4:1762.

34. Curtin SJ, Zhang F, Sander JD, Haun WJ, Starker C, Baltes NJ, et al. Targeted mutagenesis of duplicated genes in soybean with zinc-finger nucleases. Plant Physiol. 2011;156:466-73.

35. Zhang Y, Zhang F, Li X, Baller JA, Qi Y, Starker CG, et al. Transcription activator-like effector nucleases enable efficient plant genome engineering. Plant Physiol. 2013;160:20-7. 